\title{
Gross Chromosomal Rearrangements in Kluyveromyces marxianus Revealed by Illumina and Oxford Nanopore Sequencing
}

\author{
Lin Ding ${ }^{1}$, Harrison D. Macdonald ${ }^{1, \dagger}{ }^{\dagger}$ Hamilton O. Smith ${ }^{1,2}$, Clyde A. Hutchison III ${ }^{1}$, \\ Chuck Merryman ${ }^{1,+}$, Todd P. Michael ${ }^{1,+}{ }^{+}$, Bradley W. Abramson ${ }^{1,+}$, Krishna Kannan ${ }^{2}$, \\ Joe Liang ${ }^{2,+}$, John Gill ${ }^{2}$, Daniel G. Gibson ${ }^{1,2}$ and John I. Glass ${ }^{1, * \mathbb{D}}$ \\ 1 J. Craig Venter Institute, 4120 Capricorn Lane, La Jolla, CA 92037, USA; linding@usc.edu (L.D.); \\ harrison.macdonald01@gmail.com (H.D.M.); hsmith@jcvi.org (H.O.S.); chutchison@jcvi.org (C.A.H.III); \\ chuckmerryman@gmail.com (C.M.); toddpmichael@gmail.com (T.P.M.); theabramson@gmail.com (B.W.A.); \\ dan@codexdna.com (D.G.G.) \\ 2 Codex DNA, 9535 Waples St \#100, San Diego, CA 92121, USA; krishna@codexdna.com (K.K.); \\ madonjoe@gmail.com (J.L.); john@codexdna.com (J.G.) \\ * Correspondence: jglass@jcvi.org; Tel.: +1-858-200-1856 \\ + These authors contributed equally to this work.
}

Received: 30 August 2020; Accepted: 22 September 2020; Published: 26 September 2020

\begin{abstract}
Kluyveromyces marxianus (K. marxianus) is an increasingly popular industrially relevant yeast. It is known to possess a highly efficient non-homologous end joining (NHEJ) pathway that promotes random integration of non-homologous DNA fragments into its genome. The nature of the integration events was traditionally analyzed by Southern blot hybridization. However, the precise DNA sequence at the insertion sites were not fully explored. We transformed a PCR product of the Saccharomyces cerevisiae URA3 gene (ScURA3) into an uracil auxotroph K. marxianus otherwise wildtype strain and picked 24 stable Ura+ transformants for sequencing analysis. We took advantage of rapid advances in DNA sequencing technologies and developed a method using a combination of Illumina MiSeq and Oxford Nanopore sequencing. This approach enables us to uncover the gross chromosomal rearrangements (GCRs) that are associated with the ScURA3 random integration. Moreover, it will shine a light on understanding DNA repair mechanisms in eukaryotes, which could potentially provide insights for cancer research.
\end{abstract}

Keywords: gross chromosomal rearrangements; non-homologous end joining; translocation; Illumina MiSeq; Oxford Nanopore; Kluyveromyces marxianus; Saccharomyces cerevisiae; URA3 gene

\section{Introduction}

Kluyveromyces marxianus is a thermotolerant yeast [1] and is the fastest-growing eukaryote identified to date [2]. It has many other physiological features that the conventional yeasts, such as Saccharomyces cerevisiae, are lacking. As a result, K. marxianus is becoming a potentially valuable industrial yeast. Therefore, many molecular tools [3-7] have been developed for its genetic engineering. Among these are many that harness the power of the homologous recombination (HR) pathway, which requires homologous flanking sequences, for targeted gene editing. However, similar to its cousin, Kluyveromyces lactis [8], K. marxianus also embraces a robust non-homologous end joining (NHEJ) pathway in the absence of homology. It can be transformed with non-homologous DNA fragments by illegitimate recombination (IR) that involves the NHEJ pathway [9]. In the resulting transformants, the DNA fragments insert at various chromosomal locations. These two modes exist with various frequencies in different yeast strains $[10,11]$. 
Kegel et al. [8] reported genome-wide IR insertion events in Kluyveromyces lactis that were strongly biased toward intergenic regions. If they induced ectopic double strand breaks (DSBs) using restriction cleavage, then there was no bias for genic versus intergenic sequence, suggesting that genome-wide IR occurred at spontaneous mitotic DSBs that are preferentially distributed in intergenic sites. Nonklang et al. [12] used a PCR product of the Saccharomyces cerevisiae URA3 (ScURA3) gene to transform a K. marxianus DMKU3-1042 ura3 $\triangle$ mutant. They analyzed 9 Ura+ transformants by Southern blot hybridization. The ScURA3 insertions were in different genome locations and one transformant had multiple insertions. Abdel-Banat et al. [9] reported very high frequency insertion

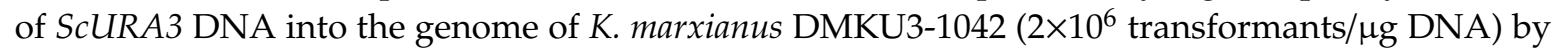
the NHEJ pathway. They suggested that a high-density ScURA3 insertion map analogous to that obtained by global transposon mutagenesis $[13,14]$ could be generated. In order to fully understand the NHEJ repair mechanism in K. marxianus, it is desired to have the precise sequence at the junctions of insert sites in the genome. This is not possible to be achieved by Southern blot hybridization due to its low resolution, not to mention its laboriousness.

We generated our own Ura+ K. marxianus transformants by transforming a 1123-bp PCR product of the Saccharomyces cerevisiae URA3 gene (ScURA3) and took advantage of Illumina MiSeq and whole-genome Oxford Nanopore sequencing to produce a detailed analysis of 24 Ura + transformant clones. We found a surprising variety of insertion events. In addition to three tandem dimer and two trimer ScURA3 concatemer insertions, two insertions produced inversions, one produced a large deletion, and another three Ura+ transformants produced chromosomal translocations in which each end of ScURA3 were inserted in a different chromosome. Sequencing analysis, especially the long reads obtained with Nanopore sequencing, was instrumental in detecting and analyzing GCRs that are a hallmark of cancers. Therefore, our results and method may provide insights to understanding the basic mechanisms of DNA repair and cancer biology.

\section{Results}

\subsection{Determination of ScURA3 Genomic Insertion Sites by Illumina MiSeq Analysis}

We constructed two separate libraries containing either ScURA3 head or tail junctions (Figure 1). Illumina MiSeq 100-bp sequences from the head library were scanned for ScURA3 head sequence matches followed by at least $20 \mathrm{bp}$ of the K. marxianus sequence. This $K$. marxianus sequence identified the chromosome and the site of a ScURA3 insertion. Similarly, ScURA3 tail junctions were identified. In total, we identified 23 head and 23 tail genome junctions (Figure 2). If head and tail junctions were very close on the same chromosome, they were paired and assumed to be produced by a single ScURA3 insertion event. This could then be confirmed by PCR using primers designed from the flanking $K$. marxianus sequences (Table S1). Testing all the clones with a given primer set will point to the specific clone carrying the ScURA3 insert.

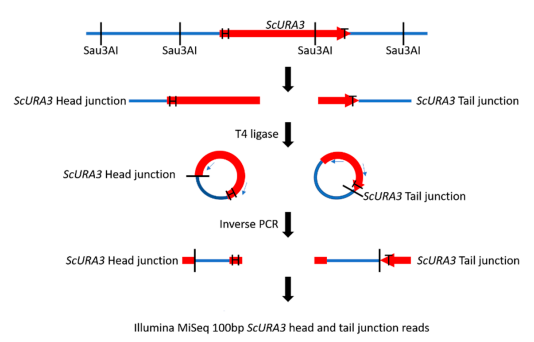

Figure 1. Construction of ScURA3 head (arrow, labeled H) and tail (labeled T) junction libraries. DNA from a SCURA3 transformant clone is cleaved at GATC sites with Sau3AI and the fragments are ligated under dilute conditions to yield DNA circles with separate ScURA3 head and tail junctions. The ligated DNA is then divided into two aliquots. Circles containing the head junctions are amplified in a PCR reaction with primers ura $3+61 \mathrm{c}$ and ura3 TestF. The tail junctions are amplified with ura $3+720 \mathrm{c}$ and ura3 R1-1-DN primers. The result is separate head and tail junction libraries. 


\begin{tabular}{|c|c|c|c|c|c|}
\hline & K. marxianus genome & ScURA3 Head & $\mathrm{Chr}$ & Location & Method \\
\hline 1 & GGAATTTGCCAAGTCCAGAGGTAT & TGAGAGTGCACCACGCTTTTCAA & 1 & 397946 & MiSeq \\
\hline 2 & CTCTATATCATTAAAGAGCCTG & tgagagtgCACCACGCTTTTCAA & 1 & 1128870 & MiSeq \\
\hline 3 & TCTTCAATACCAAATTCGTCGTCTAGAG & TGAGAGTGCACCACGCTTTTCAA & 2 & 628004 & MiSeq \\
\hline 4 & TTCAAAGTGGAAAAAAACACCA & TGAGAGTGCACCACGCTTTTCAA & 4 & 1364429 & MiSeq \\
\hline 5 & TAACTTCGGAATATTGAATTAATCGTCTCC & TGAGAGTGCACCACGCTTTTCAA & 7 & 24526 & MiSeq \\
\hline 6 & ACCAAAAAAAACCATAAAAACCAAAACA & TGAGAGTGCACCACGCTTTTCAA & 4 & 714164 & MiSeq \\
\hline 7 & ATCTTGAAGAGGAAAAGCATACCA & TGAGAGTGCACCACGCTTTTCAA & 3 & 953765 & Miseq \\
\hline 8 & TCCAAAGTCAAACCTTCCAAGTCGACG & TGagAGTGCACCACGCTTTTCAA & 1 & 377332 & Miseq \\
\hline 9 & TGTACTTCTACGAAGGGCCAAGGCC & TGAGAGTGCACCACGCTTTTCAA & 7 & 185317 & Miseq \\
\hline 10 & TGTGGCTAAGGTAACGGCAAGC & TGAGAGTGCACCACGCTTTTCAA & 2 & 1256630 & MiSeq \\
\hline 11 & CCCTGCCGTATATACCTGAAAGTTGATTTA & TGAGAGTGCACCACGCTTTTCAA & 5 & 525553 & MiSeq \\
\hline 12 & GATGATGATGATAAGATAGTGAAGAAGG & TGAGAGTGCACCACGCTTTTCAA & 6 & 166286 & MiSeq \\
\hline 14 & GACCTCGGGGCGTCGGGGTAACTA & TGAGAGTGCACCACGCTTTTCAA & 6 & 392781 & MiSeq \\
\hline 15 & АAATTATTATGGAACAATTTGTGTGATTA & tgagagtgcaccaCGCTTTTKAA & 1 & 1098288 & MiSeq \\
\hline 16 & GCCGTAACCTCTTTTITAATTGCCAC & TGAGAGTGCACCACGCTTTTCAA & 3 & 718057 & Miseq \\
\hline 17 & GATTTAATTAATATATATTATATAACAAC & TGAGAGTGCACCACGCTTTTCAA & 1 & 560632 & Miseq \\
\hline 18 & CATTAGCACCAAACCCACGGAAATTCCTG & TGAGAGTGCACCACGCTTTTCAA & 5 & 821611 & Miseq \\
\hline 19 & СCTATTGAGAAAAAAACCGAAACTATTT & TGAGAGTGCACCACGCTTTTCAA & 5 & 1339373 & MiSeq \\
\hline 20 & ACTTCTAAATCGGATAGATTCGAGGT & TGAGAGTGCACCACGCTTTTCAA & 8 & 866469 & Miseq \\
\hline 21 & TTTATTTTAGTGTTTTATTG & TGAGAGTGCACCACGCTTTTCAA & 1 & 926450 & Nanopore \\
\hline 22 & TGAAAAAATGATCCTGAAAAGA & TCAGAGTGCACCACGCTTTTCAA & 2 & 826489 & MiSeq \\
\hline 23 & TATACACGAGATATTAGTCGAGTGGGA & GTCCCATGCACCACGCTTTTCAA & 4 & 101355 & MiSeq \\
\hline 24 & CATGCCGGCCGAGTAACCGAGAGAA & TGAGAGTGCACCACGCTTTTCAA & 1 & 1643311 & MiSeq \\
\hline
\end{tabular}

(A). Head junctions

\begin{tabular}{|c|c|c|c|c|c|}
\hline lone \# & ScURA3 Tail & K. marxianus genome & $\mathrm{Chr}$ & Location & Method \\
\hline 1 & СААТTТААТTAТАТСАGТТАТTАСССТG & GGGCAGCAGTAGTGGATATGCAGC & 1 & 397947 & Nanopore \\
\hline 2 & CAATTTAATTATATCAGTTATTACCCTG & TCTATCAGGGTCGAATCATCGCATC & 1 & 1128881 & MiSeq \\
\hline 3 & CAATTTAATTATATCAGTTATTACCCT $g$ & CCTTCACCAAAGGAGCCAAACAA & 2 & 628003 & MiSeq \\
\hline 4 & CAATTTAATTATATCAGTTATTACCCtg & CGTTAGATAATCCTGTGAAATCGT & 4 & 1364423 & MiSeq \\
\hline 5 & CAATTTAATTATATCAGTTATTACCCtg & TATATTATATTAAATAAAAATAAT & 6 & 375003 & MiSeq \\
\hline 6 & САATTTAATTATATCAGTTATTACCCTG & AACGACTTGATGATGTACATAG & 4 & 762150 & MiSeq \\
\hline 7 & CAATTTAATTATATCAGTTATTACCCTG & ATGCTGGCATGAGGGGGGGAAGTC & 3 & 824416 & MiSeq \\
\hline 8 & CAATTTAATTATATCAGTTATTACCCTG & GTAATGCAAATTTATACAGGTCAAA & 1 & 375438 & MiSeq \\
\hline 9 & CAATTTAATTATATCAGTTATTACCCTG & AGCTTTTGAGAAAAAAAAGTTTTA & 7 & 185307 & MiSeq \\
\hline 10 & CAATTTAATTATATCAGTTATTACCCTG & TTGGCGCCAGGTCACTTGGT & 2 & 1256656 & MiSeq \\
\hline 11 & CAATTTAATTATATCAGTTATTACGGCT & TACGCCTCTTAAATCCCAGATC & 5 & 525567 & Miseq \\
\hline 12 & CAATTTAATTATATCAGTTATTACCCTG & AGAATCTAGTCTTGTTTGGAAAGTAAA & 6 & 166289 & MiSeq \\
\hline 14 & CAATTAATTATATCAGTTATTACCGTG & GCGGTCTGAGGGCGTITCTIITCG & 6 & 392769 & Miseq \\
\hline 15 & CAATTTAATTATATCAGTTATTACCCtg & CATACGCGAAACTCAGGTGCTGCA & 5 & 1165171 & Nanopore \\
\hline 16 & CAATTTAATTATATCAGTTATTACCCtg & TATIIIIIIIIIITICGTIIIITCCG & 3 & 718026 & MiSeq \\
\hline 17 & CAATTTAATTATATCAGTTATTACCCT $g$ & CTAAAGATACCAAGACGATAGTITG & 1 & 560631 & MiSeq \\
\hline 18 & CAATTTAATTATATCAGTTATTACCCTG & TCCTAATAAAACACCAGGTCTCAA & 5 & 821620 & MiSeq \\
\hline 19 & GGACttaattatatcagttattaccctg & AAATTCTTGACTAAATAACACACTC & 5 & 1339385 & MiSeq \\
\hline 20 & CAATTTAATTATATCAGTTATTACCCTG & GACTCAAAATTAAATGCCAGAGT & 8 & 866482 & MiSeq \\
\hline 21 & САATTTAATTATATCAGTTATTACCCTG & 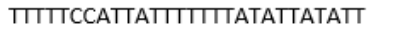 & 1 & 926445 & MiSeq \\
\hline 22 & CAATTTAATTATATCAGTTATTACCCTG & TATCAGCATCTACGTCACATGCAACACC & 2 & 826479 & MiSeq \\
\hline 23 & CAATTTAATTATATCAGTTATTACCCTG & GTATCTTCGCTTTGTCTCTTAGCTTCC & 6 & 7292 & MiSeq \\
\hline 24 & САATTTAАТTATATСAGTTATTACСCTG & TCGGATCGATTCTTATGCG & 1 & 1643296 & MiSeq \\
\hline
\end{tabular}

\section{(B). Tail junctions}

Figure 2. Head and tail junction sequences from Illumina MiSeq reads of the inverse PCR 24-clone libraries constructed as shown in Figure 1. (A). Head junctions. (B). Tail junctions. Chromosome (Chr) and insertion sites are indicated. Lower case, italicized, bold letters indicate deleted bases. Upper case, italicized, bold letters indicate base substitutions.

\subsection{Concatemer Dimer and Trimer ScURA3 Inserts}

The 24 clones were screened for the presence of URA3+ concatemers by performing PCR with the primers ura3+61c and ura3+720 (Table S1). If ScURA3 head to tail joints are present, a PCR product of $463 \mathrm{bp}$ should result. Five clones 2, 3, 5, 12, and 19 gave the expected product. In addition, PCRs 
performed with primers flanking the ScURA3 concatemers yielded products of the expect sizes. Clones 3,5 , and 19 contained dimers and clones 2 and 12 contained trimers. These PCRs also showed ladders of bands corresponding to monomers, dimers, and higher bands as expected since during the annealing step of each PCR cycle, some of the ScURA3 concatemers may hybridize out of phase (Figure S4). These results were subsequently confirmed by Oxford Nanopore sequence reads that spanned the entire concatemer and flanking sequence.

The head to tail junctions in the dimer and trimer inserts might be expected to show the effects of NHEJ repair. These junctions are readily observed in both Oxford Nanopore and Illumina MiSeq reads. Among the seven junctions, we observed only three different types: CCCTG|TGAGA, CCCtg|TGAGA, and CCATg|TGAGA, where the bold lowercase italicized letters are deleted bases and the bold uppercase italicized letters are substitutions or new bases. Note that we cannot distinguish whether the $t g$ in the second junction type is deleted from the tail as shown, or from the head.

\subsection{Sixteen ScURA3 Insertion Events Were Either Precise or Resulted in Small Deletions of Genome Sequence}

Sixteen clones appeared to be simple events that involved insertion of ScURA3 into a single break in the K. marxianus genome (Table 1). Two of the clones ( 3 and 17) contained precise ScURA3 insertions, that is, no loss of genome sequence occurred at the insertion site. In both cases, the ScURA3 terminal head sequences were unaltered; however, there was a loss of the terminal $\mathrm{G}$ of the tail sequence in both cases. In 14 clones $(2,4,9,10,11,12,14,16,18,19,20,21,22$, and 24), insertions were accompanied by small deletions of the genome sequence, ranging from 2 to $30 \mathrm{bp}$. Several of these also involved alterations of the terminal head or tail sequences at the junctions. In clone 19, the ScURA3 tail had lost 24 terminal bases followed by 4 base substitutions (Figure 2). The observations are compatible with DSB repair by the error-prone NHEJ pathway [15]. 
Table 1. Types of ScURA3 insertion events. The asterisk next to clones 1 and 13 indicates incomplete information (see text). Chr: Chromosome. \#: number (see text). ?: unknown.

\begin{tabular}{|c|c|c|c|c|c|c|c|c|}
\hline \multirow{2}{*}{$\frac{\text { Clone \# }}{1^{*}}$} & \multirow{2}{*}{$\begin{array}{c}\text { Primer Set } \\
\text { none }\end{array}$} & \multirow{2}{*}{$\frac{\text { Chr }}{1}$} & \multirow{2}{*}{$\begin{array}{c}\text { Head Junction Location } \\
397946\end{array}$} & \multirow{2}{*}{$\begin{array}{c}\text { Tail Junction Location } \\
397947\end{array}$} & \multicolumn{2}{|c|}{$\begin{array}{c}\text { Type of Insertion } \\
\text { and Base Pair Involved }\end{array}$} & \multirow{2}{*}{$\begin{array}{l}\text { ScURA3 } \\
\text { monomer }\end{array}$} & \multirow{2}{*}{$\begin{array}{c}\text { Gene or Intergenic Insertion } \\
\text { SG4EUKG585063 (ADE1) }\end{array}$} \\
\hline & & & & & precise & $0 \mathrm{bp}$ & & \\
\hline 3 & 2 & 2 & 628004 & 628003 & precise & $0 \mathrm{bp}$ & dimer & SG4EUKG585526 \\
\hline 17 & 16 & 1 & 560632 & 560631 & precise & $0 \mathrm{bp}$ & monomer & intergenic \\
\hline 2 & 1 & 1 & 1128870 & 1128881 & del & $10 \mathrm{bp}$ & trimer & intergenic \\
\hline 4 & 26 & 4 & 1364429 & 1364423 & del & $5 \mathrm{bp}$ & monomer & SG4EUKG587507 \\
\hline 9 & 37 & 7 & 185317 & 185307 & del & $9 \mathrm{bp}$ & monomer & intergenic \\
\hline 10 & 11 & 2 & 1256630 & 1256656 & del & $25 \mathrm{bp}$ & monomer & intergenic \\
\hline 11 & 21 & 5 & 525553 & 525567 & del & $13 \mathrm{bp}$ & monomer & intergenic \\
\hline 12 & 4 & 6 & 166286 & 166289 & del & $2 \mathrm{bp}$ & trimer & intergenic \\
\hline $13 *$ & none & $?$ & $?$ & $?$ & $?$ & $?$ & monomer & $?$ \\
\hline 14 & 20 & 6 & 392781 & 392769 & del & $11 \mathrm{bp}$ & monomer & intergenic \\
\hline 16 & 25 & 3 & 718057 & 718026 & del & $30 \mathrm{bp}$ & monomer & intergenic \\
\hline 18 & 27 & 5 & 821611 & 821620 & del & $8 \mathrm{bp}$ & monomer & SG4EUKG588009 \\
\hline 19 & 3 & 5 & 1339373 & 1339385 & del & $11 \mathrm{bp}$ & dimer & intergenic \\
\hline 20 & chr8 & 8 & 866469 & 866482 & del & $12 \mathrm{bp}$ & monomer & SG4EUKG589280 \\
\hline 21 & 32 & 1 & 926450 & 926445 & del & $4 \mathrm{bp}$ & monomer & intergenic \\
\hline 22 & 12 & 2 & 826489 & 826479 & del & $9 \mathrm{bp}$ & monomer & SG4EUKG585988 \\
\hline 24 & 17 & 1 & 1643311 & 1643296 & del & $14 \mathrm{bp}$ & monomer & intergenic \\
\hline 6 & 9 & 4 & 714164 & 762150 & inversion & $47,986 \mathrm{bp}$ & monomer & intergenic-intergenic \\
\hline 7 & 7 & 3 & 953765 & 824416 & del & $129,349 \mathrm{bp}$ & monomer & intergenic-intergenic \\
\hline 8 & 5 & 1 & 377332 & 375438 & inversion & $1892 \mathrm{bp}$ & monomer & SG4EUKG584656-intergenic \\
\hline 5 & $\mathrm{cl} 5$ & $7-6$ & $24526(7)$ & $375003(6)$ & Translocation & Translocation & dimer & intergenic-intergenic \\
\hline 15 & 10 & $1-5$ & 1098288(1) & $1165171(5)$ & Translocation & Translocation & monomer & intergenic-intergenic \\
\hline 23 & 8 & $4-6$ & $101355(4)$ & $7292(6)$ & Translocation & Translocation & monomer & SG4EUKG586913-intergenic \\
\hline
\end{tabular}




\subsection{Two ScURA3 Insertion Events Were Simple Insertions but Involved Corruption of the ScURA3 Termini}

Clone 1 has an insertion in the ade1p gene at position 397,946 on chromosome 1, which is in the open reading frame of (SG4EUKG585063, homologous to ScADE1). As a result of the disruption of the ADE1 gene, K. marxianus colonies are pink on low adenine medium. This is consistent with Ade mutants in other yeasts. It appeared to be precise except for an inserted $65 \mathrm{bp}$ DNA sequence between the ScURA3 tail junction and the genome sequence. This DNA did not convincingly match any of the genome sequence. Its source is not known. It seems unlikely, because of its length, to have been inserted by an NHEJ polymerase not requiring a template. One result of this insertion was the inability to identify a tail junction sequence for clone 1 in the MiSeq data. The head junction sequence was readily identified. Only by Oxford Nanopore sequencing was the insertion detected and the point of insertion of ScURA3 DNA into the genome identified.

In clone 13, about $20 \mathrm{bp}$ of the ScURA3 tail sequence and $78 \mathrm{bp}$ of the head sequence were missing, thus there were no junction sequences in Figure 2 and the chromosome location of the insert was not found.

\subsection{Three ScURA3 Insertions Resulted in Large Chromosomal Inversions or Deletions}

Clone 6 produced an inversion of the genomic segment between 2 DSBs 47,986 bp apart on chromosome 4 (Table 1 and Figure 3). Oxford Nanopore reads were necessary to solve the structure (Figure S5). Each DSB produces two ends, and these can be labeled 1 and 2 for the first break, and 3 and 4 for the second break (Figure 3A). ScURA3 DNA has two ends labeled H and T. Thus, several possible repair reactions can occur. Clone 6 exhibited 1H-T3 joining followed by 2-4 joining, resulting in inversion of the 2-3 segment. Contour-clamped homogeneous electric field (CHEF) electrophoresis gel analysis of this clone was identical to the wild type (Figure 4).
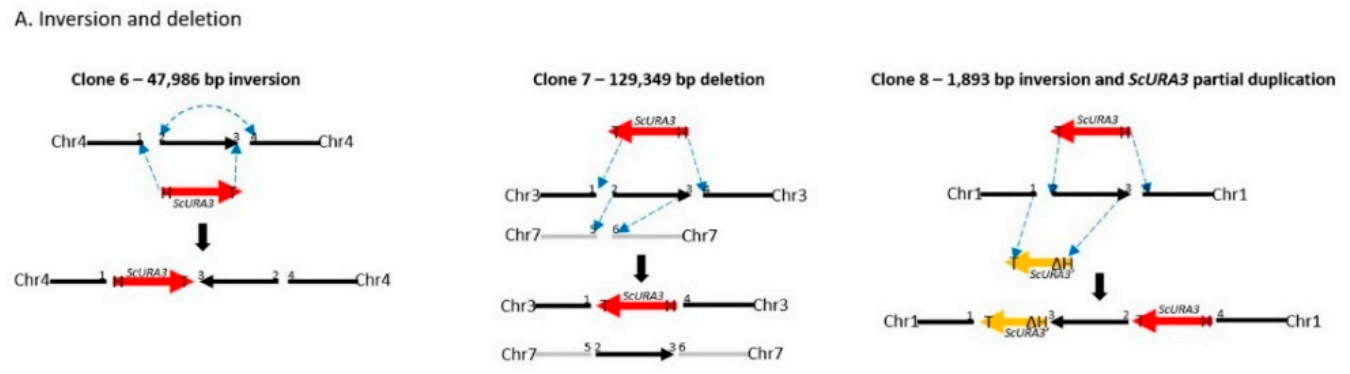

B. Translocation
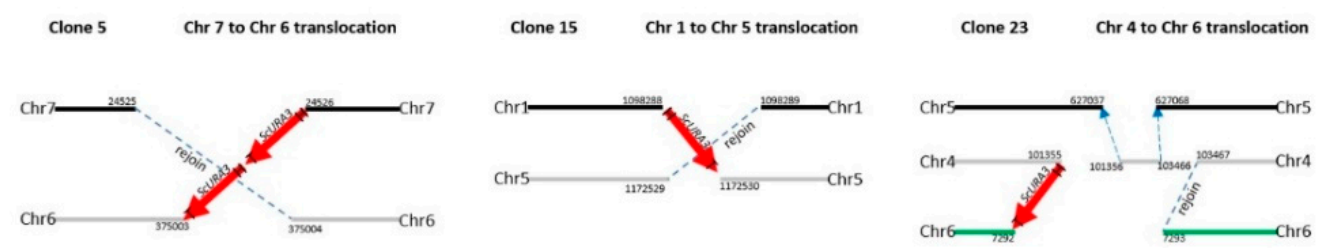

Figure 3. Diagrams of the structures of the ScURA3 insertion events revealed from Illumina MiSeq and Oxford Nanopore sequencing analysis. (A). Inversion and deletion events observed in clone 6, 7, and 8. (B) Translocation events observed in clone 5, 15, and 23. 

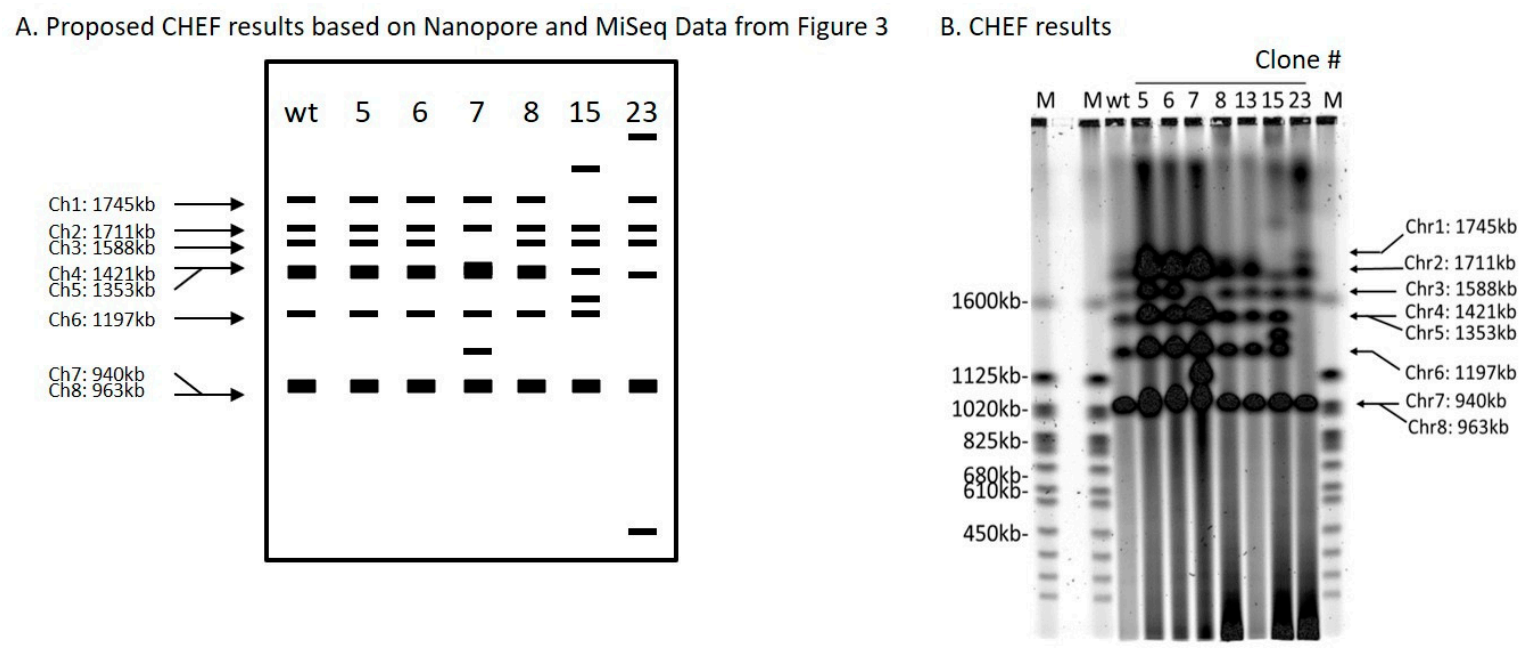

Figure 4. Contour-clamped homogeneous electric field (CHEF) analysis of ScURA3 inversion and deletion as well as translocation events. (A). A schematic of expected band patterns of each clone. (B). CHEF results of clone $5,6,7,8,13,15$, and 23 .

Clone 7 involved 2 DSBs in chromosome 3 and one DSB in chromosome 7. First, a 129,349 bp 2-3 segment was deleted from chromosome 3 in a 1T-H4 ScURA3 insertion reaction. This was followed by insertion of the $2-3$ segment at a third DSB in chromosome 7 (Table 1, Figure 3A, and Figure S6). Thus, it is predicted that chromosome 7 will increase from 940 to $1070 \mathrm{~kb}$ and chromosome 3 will decrease from 1588 to $1458 \mathrm{~kb}$ as confirmed by CHEF gel analysis (Figure 4).

The clone 8 insertion is more complex. In this case, the DSBs were $1893 \mathrm{bp}$ apart on chromosome 1 and ScURA3 integration involved 2T-H4 joining, resulting in an inversion of the 1893-bp segment. The 1 and 3 ends then interacted with another ScURA3 sequence, yielding the inverted 1892-bp segment separating the two ScURA3s. Furthermore about $200 \mathrm{bp}$ of the head of the second ScURA3 is missing. The overall structure is thus $1 \mathrm{~T}-\Delta \mathrm{H} 3-2 \mathrm{~T}-\mathrm{H} 4$ (Figure $3 \mathrm{~A}$ ). Several Oxford Nanopore reads confirm this structure both from the 24-clone pool and from the barcoded clone 8 reads (Figure S7). The CHEF gel chromosome banding pattern was wild type as expected (Figure 4).

\subsection{Three ScURA3 Insertions Resulted in Inter-Chromosomal Translocations}

For clones 5, 15, and 23, Oxford Nanopore reads located the ScURA3 head and tail junctions on separate chromosomes, indicating translocation events (Table 1, Figures S8-S10). In clone 5, a ScURA3 dimer inserted to produce a reciprocal exchange between the arms of chromosomes 7 and 6 (Figure S8) as diagrammed in Figure 3B. One pair of arms is bridged by the ScURA3 dimer. The nature of the rejoin by the other two arms has not been determined. The CHEF gel bands are similar to wild type (Figure 4).

Clone 15 contained a ScURA3 monomer insert with one end in chromosome 1 and the other in chromosome 5 (Figure S9). The ScURA3 tail junction in chromosome 5 falls in a region of repetitious sequence making the exact location uncertain. However, K. marxianus assembly based on Oxford Nanopore reads gives a single location in chromosome 5. The rejoin of chromosome 1 and 5 was not successfully located in the Oxford Nanopore reads. Chromosome 5 is predicted to decrease from 1353 to $1279 \mathrm{~kb}$ while chromosome 1 should increase from 1745 to $1819 \mathrm{~kb}$ (Figure 4 ).

Clone 23 contained a ScURA3 insert with one end in chromosome 4 and the other in chromosome 6. The translocation is interesting in that the short ends of chromosome 4 and 6 are joined to yield a new short chromosome (Figure 3B and Figure S10). Rejoin of the long arms occurs at positions 7293 and 103467 to yield an extra-long chromosome. In addition, a short piece of chromosome 4 is released and integrates into chromosome 5 at approximately position 627037 (Figure 3B). Bands 4 and 6 disappear, while the short and very long new chromosomes are not identifiable on the CHEF gel (Figure 4). Interestingly, the band for chromosome 5 also seems to have disappeared. 


\section{Discussion}

During transformation of K. marxianus, there is typically a 50- to 100-fold excess of ScURA3 DNA molecules to yeast cells. It is expected that many cells will take up several ScURA3 molecules. The NHEJ pathway proteins, Ku70 [9], and Ku80 [6] are required for production of Ura+ in K. marxianus. NHEJ proteins act on the free ScURA3 ends, resulting in monomer circles as well as tandem concatemers. The probability of these different events is not known, but it seems likely that free unreacted ScURA3 ends would not persist for long. Proximity of ends to each other probably determines how likely a pair of ends are to react. However, if endogenous DSBs are simultaneously present in the genome of the same cell, these would be expected to occasionally join to free ScURA3 ends to yield Ura+ transformants. This is a rare event leading to only a few thousand transformants among the millions of K. marxianus cells present per transformation reaction. By plating on CAA-U plates, insertion of ScURA3 DNA into the genome is specifically selected for since non-inserted ScURA3 DNA does not independently replicate and is diluted out among the progeny cells. To discover the types of insertion events that might occur, we isolated 24 Ura+ clones for detailed study.

We developed a method using a combination of Illumina MiSeq and Oxford Nanopore sequencing analysis to reveal the precise nucleotide sequence right at the junction of ScURA3 random insertion sites, instead of using the laborious Southern blot hybridization. We found that for certain insertions, only long-read Nanopore sequencing is capable of resolving the new structure. Our analysis showed that three clones contained dimer inserts and two were trimers. The rest were monomer inserts. Eighteen events involved simple insertion into a single DSB in the K. marxianus genome while six involved two or more DSBs. Three events produced translocations in which the two ends of the ScURA3 cassette inserted into different chromosomes. Two of the latter events produced inversion of DNA between the two DSBs and one produced a large deletion in which the 129-kb deleted segment reinserted into another chromosome. Interestingly, no notable growth defects were observed in these clones even for clone 7 that lost $129 \mathrm{~kb}$ of genetic material. This indicates the elasticity that K. marxianus genome has. Further investigation may uncover DNA repair mechanisms that could be valuable to understanding chromosome evolution and therefore provide insight to speciation and cancer biology

\section{Materials and Methods}

\subsection{Yeast Strains}

K. marxianus NRRL Y-6860 was obtained from the U.S. Department of Agriculture Agricultural Research Service Culture Collection. We sequenced the genomic DNA and identified 8 chromosomes comprising the 10,837,618-bp genome, and 4963 genes were identified (GenBank number GCA_002356615.1). Strain G13 (ura3 $\Delta$ ) was constructed as in Figure S1. The KmURA3 ORF in K. marxianus NRRL Y-6860 was removed by homologous recombination (HR)-mediated 5-FOA counter selection. Primers used for strain construction and confirmation are listed in Table S1.

\subsection{Preparation of ScURA3 Cassette DNA}

The ScURA3 cassette contained in the plasmid pRS316 (ATCC ${ }^{\circledR} 77145^{\mathrm{TM}}$; Manassas, VA, USA) was PCR-amplified using the two primers $5^{\prime}$-tgagagtgcaccacgctttcaattc and $5^{\prime}$-cagggtaataactgatataattaaattg. The 5' OH PCR product (1123 bp, Figure S2) was purified using the QIAquick PCR purification kit (Qiagen, Hilden, Germany). For purposes of calculation, $1 \mu \mathrm{g}$ of ScURA3 DNA contains approximately $10^{12}$ molecules. For convenience, the $5^{\prime}$ end of ScURA3 is called the "head" and the $3^{\prime}$ end is the "tail" (Figure 1).

\subsection{Isolation of K. marxianus ScURA3 Transformants and Preparation of Transformant DNAs}

Transformation buffer (TFB) consists of 9 parts PEG/Li acetate solution $(20 \mathrm{~mL}$ of $60 \%$ polyethylene glycol 3350 (MilliporeSigma 202444, Burlington, MA, USA), $1.5 \mathrm{~mL}$ of $4 \mathrm{M}$ lithium acetate, and $5.5 \mathrm{~mL}$ of sterile water) and 1 part of fresh $1 \mathrm{M}$ dithiothreitol. For transformation, K. marxianus cells were 
grown for $24 \mathrm{~h}$ in $30 \mathrm{~mL}$ of YPD medium at $30^{\circ} \mathrm{C}$, centrifuged at $3000 \mathrm{rpm}$ for $5 \mathrm{~min}$ and resuspended in $900 \mu \mathrm{L}$ of TFB. The cell suspension was transferred to a 1.5-mL Eppendorf tube and centrifuged at $3000 \mathrm{rpm}$ for $5 \mathrm{~min}$. The supernatant was removed, and the cells were resuspended in $600 \mu \mathrm{L}$ of TFB. ScURA3 DNA (70 ng) was then mixed with $50 \mu \mathrm{L}$ of the K. marxianus cell suspension (containing approximately $10^{9}$ cells) and incubated at $42^{\circ} \mathrm{C}$ for $30 \mathrm{~min}$. In total, $100 \mu \mathrm{L}$ of CAA-U medium ( $2 \%$ glucose, $0.6 \%$ casamino acid, $25 \mu \mathrm{g} / \mathrm{mL}$ adenine $50 \mu \mathrm{g} / \mathrm{mL}$, tryptophan, and $0.67 \%$ YNB without amino acids) were added and the cells were spread on a CAA-U/2\% agar plate and incubated at $30{ }^{\circ} \mathrm{C}$ for 2 days. Then, 24 isolated colonies were picked and patched onto a YPD plate. After incubation for a day at $30^{\circ} \mathrm{C}$, the 24 patches were re-patched on a CAA-U plate and grown another day. Twenty-four $50-\mathrm{mL}$ tubes containing $10 \mathrm{~mL}$ of CAA-U medium were inoculated from the patches and grown at $30{ }^{\circ} \mathrm{C}$ for $24 \mathrm{~h}$ on a shaker. Cells were harvested from each of the cultures by centrifugation and resuspended in $200 \mu \mathrm{L}$ of P1 cocktail ( $5 \mathrm{~mL}$ of P1 solution (Qiagen, Hilden, Germany), $5 \mu \mathrm{L}$ of $14 \mathrm{M}$ $\beta$-mercaptoethanol, and $125 \mu \mathrm{L}$ of Zymolyase $20 \mathrm{mg} / \mathrm{mL}$ ) in 1.5-mL Eppendorf tubes. The cells were incubated at $37^{\circ} \mathrm{C}$ for $30 \mathrm{~min}$ followed by addition of $20 \mu \mathrm{L}$ of $3 \mathrm{M}$ sodium acetate, and extraction with an equal volume of phenol. After centrifugation, the supernatants were harvested and precipitated with 2 volumes of ethanol. The precipitates were washed with ethanol and dissolved in $200 \mu \mathrm{L}$ of TE buffer (10 Mm Tris-Cl, 1 mM Na EDTA, pH 8). The 24 transformant DNAs ranged in concentration from approximately $50 \mathrm{ng} / \mu \mathrm{L}$ to $200 \mathrm{ng} / \mu \mathrm{L}$.

In addition to the individual transformant DNAs, cells were pooled from a plate of 24 patches and extracted as above to yield 24-clone pool DNA at approximately $200 \mathrm{ng} / \mu \mathrm{L}$.

\subsection{Preparation of 24-Clone Pool DNA Libraries for Illumina MiSeq Sequencing}

The $10.8 \mathrm{Mb}$ K. marxianus genome contains approximately 37,500 Sau3AI restriction sites (5'GATC) occurring on average every $280 \mathrm{bp}$ along the genome. There is a single site in the 1123-bp ScURA3 sequence (Figure S2) that cleaves the sequence into a 918-bp left fragment and a 203-bp right fragment. The 24-clone pool DNA was digested with Sau3AI to produce fragments with 5'GATC overhangs in a reaction mixture $(50 \mu \mathrm{L})$ containing $5 \mu \mathrm{L}$ of 10X NEB 1.1 buffer (New England Biolabs, Ipswich, MA), $5 \mu \mathrm{L}$ of 24 -clone pool DNA ( $200 \mu \mathrm{g} / \mu \mathrm{L}, \sim 10^{8}$ genome equivalents), $2 \mu \mathrm{L}$ Sau3AI (10 ug/ $\left.\mu \mathrm{L}\right)$, and $38 \mu \mathrm{L}$ water. Incubation was at $37^{\circ} \mathrm{C}$ for $2 \mathrm{~h}$ followed by inactivation of Sau3AI enzyme at $65^{\circ} \mathrm{C}$ for $30 \mathrm{~min}$. A $5-\mu \mathrm{L}$ aliquot of the fragments was mixed with $20 \mu \mathrm{L}$ of $10 \times$ T4 ligase buffer (NEB), $5 \mu \mathrm{L}$ T4 ligase (400 ug/ $\mu \mathrm{L}$, New England Biolabs, Ipswich, MA), and $170 \mu \mathrm{L}$ water. Incubation was at $23^{\circ} \mathrm{C}$ for $17 \mathrm{~h}$ followed by $72{ }^{\circ} \mathrm{C}$ for $10 \mathrm{~min}$ to inactivate the ligase. Two PCR reactions were performed. The first contained $5 \mu \mathrm{L}$ of the ligated DNA, $16 \mu \mathrm{L}$ of water, $25 \mu \mathrm{L}$ of $2 \times \mathrm{Q} 5$ master mix (New England Biolabs, Ipswich, MA), and $2 \mu \mathrm{L}$ of each of the primers ura3 $+720 \mathrm{c}$ and ura3 R1-1-DN at $25 \mu \mathrm{M}$. The second PCR was the same except that the primers were ura3 $+61 \mathrm{c}$ and ura3 TestF (Table S1). PCR settings were $98^{\circ} \mathrm{C} 10 \mathrm{~s}, 55^{\circ} \mathrm{C} 20 \mathrm{~s}$, and $72{ }^{\circ} \mathrm{C} 2$ min for 30 cycles. The first PCR reaction contained ScURA3 head to K. marxianus genome junctions and the second contained the tail junctions (Figure 1). PCR product yields were 83 and $109 \mathrm{ng} / \mu \mathrm{L}$, respectively. Illumina MiSeq 100 nucleotide sequencing reads were done on the two libraries.

\subsection{Oxford Nanopore Sequencing}

Oxford Nanopore DNA sequencing of the 24-clone pool DNA was performed as described by Oxford Nanopore Technologies. It yielded $2.1 \mathrm{~Gb}$ of sequence with a mean read length of $4658 \mathrm{bp}$ and a maximum read length of 194,297 bp (Figure S3). Single Nanopore reads gave a 10-15\% base calling error rate including base deletions. However, alignment of multiple Nanopore reads and K. marxianus genome assembly yielded the sequence that was $>95 \%$ accurate compared to the MiSeq assembly. We generally relied on Illumina MiSeq data for design of primers.

In addition to Oxford Nanopore sequencing of the 24-pool DNA, each clone DNA was bar-coded and sequenced in two 12-clone pools. Reads for each clone were then collected into 24 files ranging from 21 to $827 \mathrm{Mb}$ in size and averaging $370 \mathrm{Mb}$ per clone. 


\subsection{CHEF Genomic DNA Plug Preparation}

A single colony from Clone 5, 6, 7, 8, 13, 15, and 23 out of the 24 clones was inoculated into $50 \mathrm{~mL}$ YPD and grown at $30{ }^{\circ} \mathrm{C}$ overnight. The $1 \%$ agarose plugs were prepared using CHEF Yeast Genomic DNA Plug Kit (Bio-Rad170-3593, Hercules, CA, USA) following the manufacturer's manual. The plugs were inserted into wells of $1 \% 0.5 \mathrm{X}$ TBE agarose gel and sealed with $1 \% 0.5 \times$ TBE agarose.

\subsection{Pulsed-Field Gel Electrophoresis (PFGE)}

CHEF-DR ${ }^{\circledR}$ III Pulsed Field Electrophoresis Systems (Bio-Rad, Hercules, CA, USA) was used for running PFGE with the following settings: initial switch time: $26.3 \mathrm{~s}$, final switch time: $228 \mathrm{~s}$, gradient: $6 \mathrm{~V} / \mathrm{cm}$, angle: $120^{\circ}$, temperature: $14^{\circ} \mathrm{C}$, and total time: $36 \mathrm{~h}$. Gel was stained with $0.5 \mu \mathrm{g} / \mathrm{mL}$ ethidium bromide solution in water for $30 \mathrm{~min}$ and de-stained in distilled water for $1 \mathrm{~h}$. Genomic DNA was visualized using Typhoon 9410 Variable Mode Imager.

Supplementary Materials: Supplementary materials can be found at http:/www.mdpi.com/1422-0067/21/19/7112/s1.

Author Contributions: Conceptualization, H.O.S., C.A.H.III, C.M., K.K., D.G.G., L.D. and J.I.G.; Data curation, L.D. and H.O.S.; Formal analysis, L.D., H.O.S., C.A.H.III, T.P.M., B.W.A. and J.I.G.; Funding acquisition, D.G.G. and J.I.G.; Investigation, L.D., H.D.M., C.A.H.III, B.W.A., K.K., J.G. and D.G.G.; Methodology, L.D., B.W.A., J.L. and J.G.; Project administration, D.G.G. and J.I.G.; Resources, T.P.M.; Supervision, L.D. and J.I.G.; Writing-original draft, L.D., H.O.S., C.A.H.III and K.K.; Writing-review and editing, L.D., H.O.S. and J.I.G. All authors have read and agreed to the published version of the manuscript.

Funding: This research is funded by the Defense Advanced Research Projects Agency's Biocontrol program (contract HR0011-16-0010) and the Intelligence Advanced Research Projects Activity's Finding EngineeringLinked Indicators (FELIX) program (contract N6600118C4506) for funding this work. C.A.H., D.G.G., J.G., K.K., and the J. Craig Venter Institute (JCVI) hold SGI stock and/or stock options.

Conflicts of Interest: The authors declare no conflict of interest.

\section{References}

1. Banat, I.M.; Nigam, P.; Marchant, R. Isolation of thermotolerant, fermentative yeasts growing at $52{ }^{\circ} \mathrm{C}$ and producing ethanol at $45^{\circ} \mathrm{C}$ and $50^{\circ} \mathrm{C}$. World J. Microbiol. Biotechnol. 1992, 8, 259-263. [CrossRef] [PubMed]

2. Groeneveld, P.; Stouthamer, A.H.; Westerhoff, H.V. Super life-How and why 'cell selection' leads to the fastest-growing eukaryote. FEBS J. 2009, 276, 254-270. [CrossRef] [PubMed]

3. Rajkumar, A.S.; Varela, J.A.; Juergens, H.; Daran, J.G.; Morrissey, J.P. Biological Parts for Kluyveromyces marxianus Synthetic Biology. Front. Bioeng. Biotechnol. 2019, 7, 97. [CrossRef] [PubMed]

4. Lee, M.H.; Lin, J.J.; Lin, Y.J.; Chang, J.J.; Ke, H.M.; Fan, W.L.; Wang, T.Y.; Li, W.H. Genome-wide prediction of CRISPR/Cas9 targets in Kluyveromyces marxianus and its application to obtain a stable haploid strain. Sci. Rep. 2018, 8, 7305. [CrossRef] [PubMed]

5. Lobs, A.K.; Engel, R.; Schwartz, C.; Flores, A.; Wheeldon, I. CRISPR-Cas9-enabled genetic disruptions for understanding ethanol and ethyl acetate biosynthesis in Kluyveromyces marxianus. Biotechnol. Biofuels 2017, 10, 164. [CrossRef] [PubMed]

6. Choo, J.H.; Han, C.; Kim, J.Y.; Kang, H.A. Deletion of a KU80 homolog enhances homologous recombination in the thermotolerant yeast Kluyveromyces marxianus. Biotechnol. Lett. 2014, 36, 2059-2067. [CrossRef] [PubMed]

7. Hoshida, H.; Murakami, N.; Suzuki, A.; Tamura, R.; Asakawa, J.; Abdel-Banat, B.M.; Nonklang, S.; Nakamura, M.; Akada, R. Non-homologous end joining-mediated functional marker selection for DNA cloning in the yeast Kluyveromyces marxianus. Yeast 2014, 31, 29-46. [CrossRef] [PubMed]

8. Kegel, A.; Martinez, P.; Carter, S.D.; Astrom, S.U. Genome wide distribution of illegitimate recombination events in Kluyveromyces lactis. Nucleic Acids Res. 2006, 34, 1633-1645. [CrossRef]

9. Abdel-Banat, B.M.; Nonklang, S.; Hoshida, H.; Akada, R. Random and targeted gene integrations through the control of non-homologous end joining in the yeast Kluyveromyces marxianus. Yeast 2010, 27, 29-39.

10. Maassen, N.; Freese, S.; Schruff, B.; Passoth, V.; Klinner, U. Nonhomologous end joining and homologous recombination DNA repair pathways in integration mutagenesis in the xylose-fermenting yeast Pichia stipitis. FEMS Yeast Res. 2008, 8, 735-743. [CrossRef] 
11. Cormack, B.P.; Falkow, S. Efficient homologous and illegitimate recombination in the opportunistic yeast pathogen Candida glabrata. Genetics 1999, 151, 979-987. [PubMed]

12. Nonklang, S.; Abdel-Banat, B.M.; Cha-aim, K.; Moonjai, N.; Hoshida, H.; Limtong, S.; Yamada, M.; Akada, R. High-temperature ethanol fermentation and transformation with linear DNA in the thermotolerant yeast Kluyveromyces marxianus dmku3-1042. Appl. Environ. Microbiol. 2008, 74, 7514-7521. [CrossRef] [PubMed]

13. Hutchison, C.A., 3rd; Chuang, R.Y.; Noskov, V.N.; Assad-Garcia, N.; Deerinck, T.J.; Ellisman, M.H.; Gill, J.; Kannan, K.; Karas, B.J.; Ma, L.; et al. Design and synthesis of a minimal bacterial genome. Science 2016, 351, aad6253. [CrossRef]

14. Hutchison, C.A.; Peterson, S.N.; Gill, S.R.; Cline, R.T.; White, O.; Fraser, C.M.; Smith, H.O.; Venter, J.C. Global transposon mutagenesis and a minimal mycoplasma genome. Science 1999, 286, 2165-2169. [CrossRef]

15. Lieber, M.R. The mechanism of double-strand DNA break repair by the nonhomologous DNA end-joining pathway. Annu. Rev. Biochem. 2010, 79, 181-211. [CrossRef]

(C) 2020 by the authors. Licensee MDPI, Basel, Switzerland. This article is an open access article distributed under the terms and conditions of the Creative Commons Attribution (CC BY) license (http://creativecommons.org/licenses/by/4.0/). 